\title{
COMETS
}

\section{Hello from the other side}

Astron. J. (in the press); preprint available at https://arxiv.org/abs/1903.02260

The record for the most distant discovery of an active comet belongs to comet C/2010 U3 (Boattini), observed by astronomer Andrea Boattini on 31 October 2010 at a heliocentric distance $r_{\mathrm{H}}=18.4 \mathrm{au}$. It turns out that the Canada-France-Hawaii Telescope observed it back in 2005, at pre-perihelion $\left(24.6 \leq r_{\mathrm{H}}\right.$ $\leq 25.8 \mathrm{au}$ ), with an asymmetric coma and short tail. Within 5 au of the Sun, cometary activity is driven by the sublimation of water ice, but at great distances where it is colder, different mechanisms are at play. To understand its activity, Man-To Hui and co-workers investigate the photometric and dynamical properties of C/2010 U3.

The team observed C/2010 U3 using the Keck I 10-m telescope (2011-2012) and WIYN 0.9-m telescope (2016-2018). The only way to fit the measured tail morphology is by including a Lorentz force on the dust particles, which is generally swamped by the Sun's radiation pressure for closer comets. As for surface activity, the dust grains are at least $10 \mu \mathrm{m}$ in radius, ejected at speeds less than $50 \mathrm{~m} \mathrm{~s}^{-1}$, and consistent with sublimation of $\mathrm{CO}$ and/ or $\mathrm{CO}_{2}$, in agreement with the comet's low temperatures. However, amorphous water ice could be present and, upon heating, could crystallize in an exothermic reaction, releasing trapped gases. Based on an $\mathrm{N}$-body integration analysis of 5,000 clones, the authors conclude that $\mathrm{C} / 2010 \mathrm{U} 3$ is a dynamically old Oort cloud comet and its last perihelion passage dates to $1.96 \pm$ 0.04 Myr ago. That is too far in time for the comet to retain any heat from its last visit, dispelling any connection between cometary activity and dynamical history.

\section{May Chiao}

Published online: 25 March 2019

https://doi.org/10.1038/s41550-019-0760-9 